\title{
The Pressure Field Phase in a Pump Impeller
}

\author{
Ecoulement instationnaire dans une roue d'une pompe centrifuge
}

\author{
par K.A. Kaupert \\ Zentralschweizerisches Technikum Luzern \\ T. Staubli \\ Ouestor Industries, Canada
}

On a réalisé l'étude expérimentale d'un champ de pression instationnaire dans une roue d'une pompe centrifuge à vitesse spécifique rapide $(\omega s=1,7)$ qui fonctionnait en volute à double spirale. Pour cela, on a utilisé 25 transmetteurs de pression piézorésistifs placés sur une seule aube.

L'influence de la variation de flux sur les transmetteurs de pression a été évaluée en termes de grandeur de champ de pression et de changements de phase. L'influence de l'amont du champ de pression stable de la volute a dominé le champ de pression instable dans la roue. L'information de grandeur révèle que les fluctuations de pression dans l'interaction roue-volute ont augmenté alors que l'écoulement avait dépassé le point du meilleur rendement et qu'on s'approchait du bord arrière de l'aube de la roue. La propagation du champ de pression à la vitesse du son était synchronisée avec la fréquence de passage des languettes.

\section{INTRODUCTION}

The impeller of a pump is responsible for transferring energy to a fluid flow. It performs this task through a 3D complex interaction of coriolis, inertial, pressure, and friction forces acting on the relative flow. Each of these forces are unsteady implying a greater predictability of pump behavior can be obtained with greater understanding of the impeller unsteady flow field.

A portion of this greater understanding is found here in the form of pressure field phase behavior in the impeller flow. This information essentially reveals the changing phase of blade loading in the impeller. This unsteady blade loading is the major contributor, the volute being more minor, to the pump head when the loading of all impeller blades is superposed and time averaged.

\section{II $\square$ THE TEST FACILITY AND INSTRU- MENTATION}

Under investigation was a single stage pump designed for the paper industry to transport slurry inhomogeneous substances. The impeller of outlet diameter $D_{2}=324 \mathrm{~mm}$ ran within a double spiral volute to minimize radial forces (fig. 1). The shrouded pump impeller is also shown. Geometric specifications were,

- 7 blades, shrouded impeller,

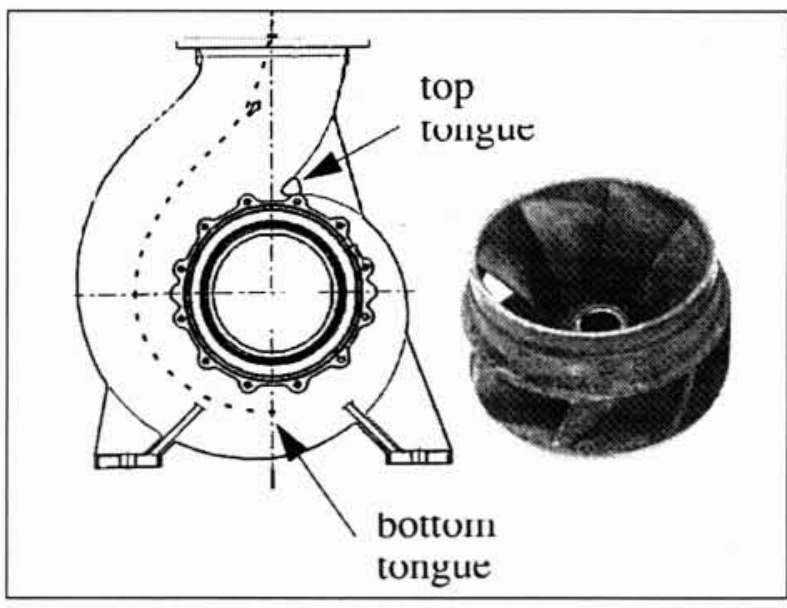

1. The double spiral volute and pump impeller.

- $D_{1} / D_{2}=0.83$ impeller inlet tip diameter,

- $B_{2} / D_{2}=0.27$ blade outlet height,

- $D_{3} / D_{2}=1.22$ volute tongues inlet diameter,

- $33^{\circ}$ blade outlet back lean angle, $20^{\circ}$ blade outlet rake.

The experiments were performed at a rotational speed of $750 \mathrm{rpm}\left(u_{2}=12.7 \mathrm{~m} / \mathrm{s}\right)$, having a best point volume flux of $0.196 \mathrm{~m}^{3} / \mathrm{s}(\phi=0.174)$ and a pressure head of 0.58 bar $(\psi=0.704)$. 
Water for the open pump circuit was drawn from a $80 \mathrm{~m}^{3}$ reservoir into the pump through a flow straightener to provide uniform inlet flow. The measurement locations in the test facility were constructed in accordance with international acceptance test norms [1].

The double spiral volute was circumferentially fitted with 32 flush mounted pressure taps, 16 on the shroud side and 16 on the hub side, near the impeller outlet at $r / R_{2}=1.05$. The resulting measured pressures were steady quantities in the stationary frame and will be presented here in nondimensional form using a dynamic pressure based on the impeller outlet tip velocity to form the steady pressure coefficient,

$$
\bar{C}_{p}=\frac{\bar{P}_{i}-\bar{P}_{1}}{0.5 \rho u_{2}^{2}}
$$

which can be interpreted as a local $\psi$ for the $i^{\text {th }}$ pressure tap since an upstream pressure difference is built.

On the impeller 25 piezoresistive pressure transducers were mounted within a single blade passage (fig. 2). Their location was selected to follow the path of two « wall streamlines " on the blade passage pressure side, Pressure Side Hub (PSH) and Pressure Side Shroud (PSS), and two "wall streamlines " on the blade passage suction side, Suction Side Hub (SSH) and Suction Side Shroud (SSS). The eigenfrequency of the transducers was known to be near 100 $\mathrm{kHz}$ in air, sufficiently high that the frequencies of interest in water, a maximum of $1 \mathrm{kHz}$, will not be detrimentally influenced. A static calibration of all transducers was performed to verify manufactures specification. Linearity was within $\pm 0.2 \%$ over the full scale of 0 to 5 bar absolute. A dynamic calibration was deemed unnecessary because of the high transducer eigenfrequency and flush mounting of the transducers. The errors in transducer measurements were quantified as $\pm 1 \%$ for the unsteady values. To reduce the transfer of any mechanical stresses from blade vibration and centrifugal forces the transducers were mounted with an elastic silicon epoxy which received detailed attention before any mounting proceeded [2].

The telemetry device was mounted on the pump shaft to send the acquired pressure transducer signals to the stationary system as a high band FM signal (fig. 3 ). Since 25 pressure transducers existed each operating point was sampled twice with 16 transducers connected. Repeatability was confirmed with 7 channels measured redundantly. These

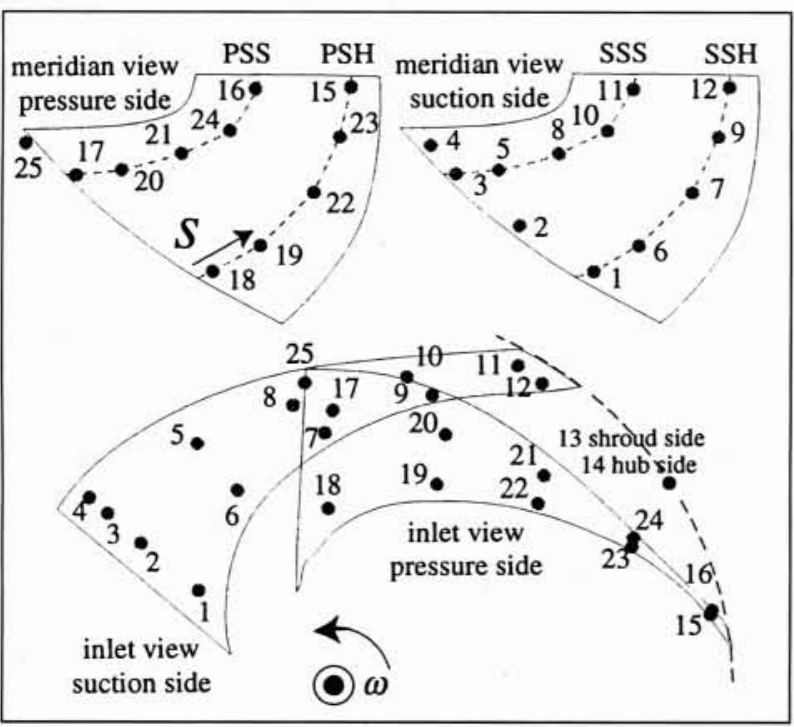

2. The location in the meridian and inlet view of the pressure transducers.

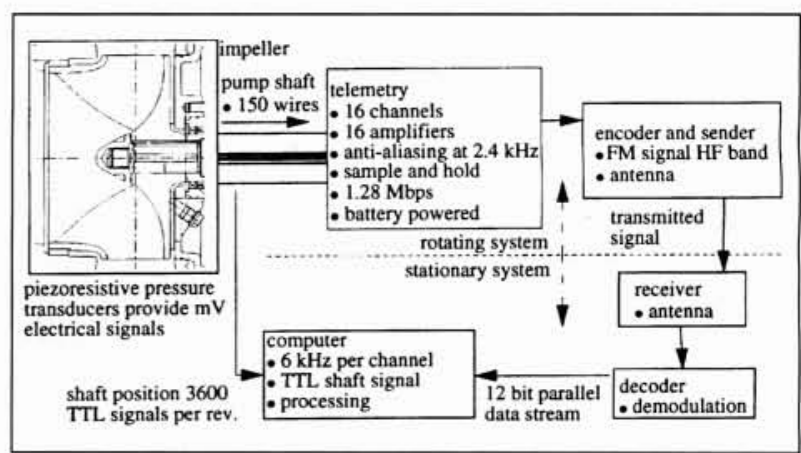

3. Telemetry data acquisition from rotating system.

measurements are unsteady quantities in the impeller frame and will be presented here in nondimensional form as the unsteady pressure coefficient,

$\tilde{C}_{p}=\frac{\tilde{P}_{i}}{0.5 \rho u_{2}^{2}}$,

and the total pressure coefficient, $C_{p}=\bar{C}_{p}+\tilde{C}_{p}$.

All signal analysis was performed for a sampling set of $2^{17}$ points per channel, slightly more than 270 rotations. The signals were phase averaged using a single shaft position provided by the shaft position encoder. This defined a starting point for all analysis to allow phase velocity calculations between transducers.

\section{III — VOLUTE INFLUENCE. STATIONARY SYSTEM}

The pump impeller and the double spiral volute form a matching of the angular momentum exchange to determine a best efficiency point $\phi$ (bep). The pressure discharge characteristic for the pump and the impeller [3] is shown in figure 4 with an accompanying calculated volute head line based on estimation of the angular momentum in the volute [4]. This calculation uses the volute throat area, the assumption of free vortex flow in the volute, and the continuity equation to determine $c_{u 2}$. Any mismatch in the angular momentum exchange causes the flow in the volute to either be accelerated for $\phi>\phi_{\text {bep }}$ or decelerated for $\phi<\phi_{\text {bep }}$.

This simplistic models merit can be used to interpret the circumferential pressure distribution of the volute wall pressure taps at $r / R_{2}=1.05$ for four volume fluxes (fig. 5). At

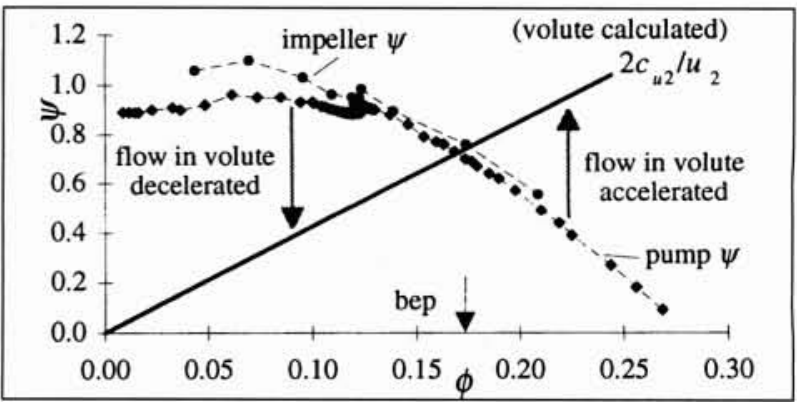

4. Pump characteristic with calculated representation of angular momentum exchange between impeller and volute. 


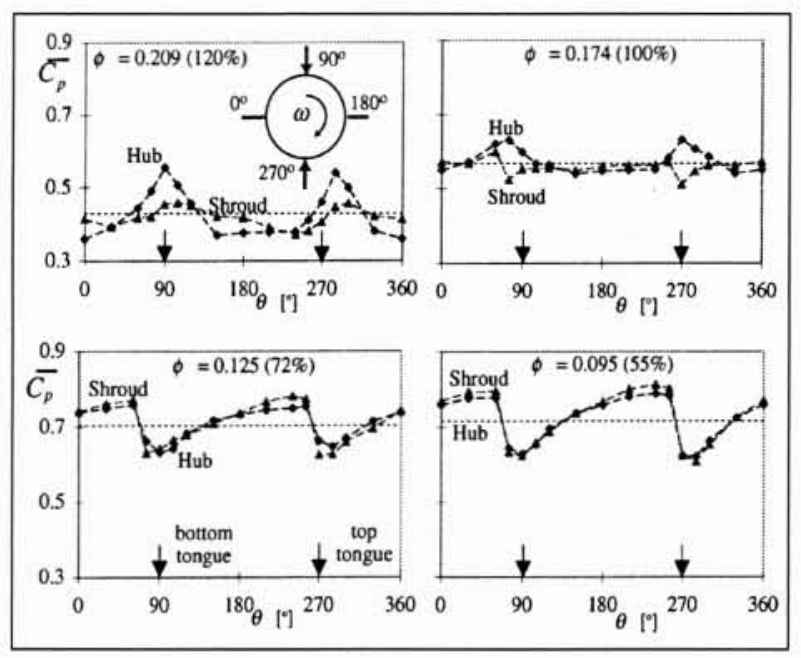

5. The circumferential pressure distribution at for volume fluxes.

operating points $\phi<\phi_{\text {bep }}$ the value of $C_{p}$ can be seen to rise in the direction of impeller blade rotation with the two volute tongues acting as boundaries to separate the volute flow into two distinct halves. The volute flow was decelerated as the simplistic model predicts. At $\phi_{\text {bep }}$ the variation in $C_{p}$ was smaller while at $\phi>\phi_{\text {bep }}$ a reverse tendency to part load was exhibited in the distribution. The volute flow was accelerated as the simplistic model predicts. This was in accordance with previously reported experimental results $[5,6]$ for single spiral volutes.

\section{V VOLUTE INFLUENCE. ROTATING SYSTEM}

Interpretation of the unsteady pressure field within the impeller must be made keeping the results of figure 5 in mind. They represent the average volute pressure a blade passage interacts with as it passes a particular point in the volute. The stationary frame pressure distribution is experienced as an unsteady pressure distribution in the impeller frame. Missing from these measurements is the unsteady

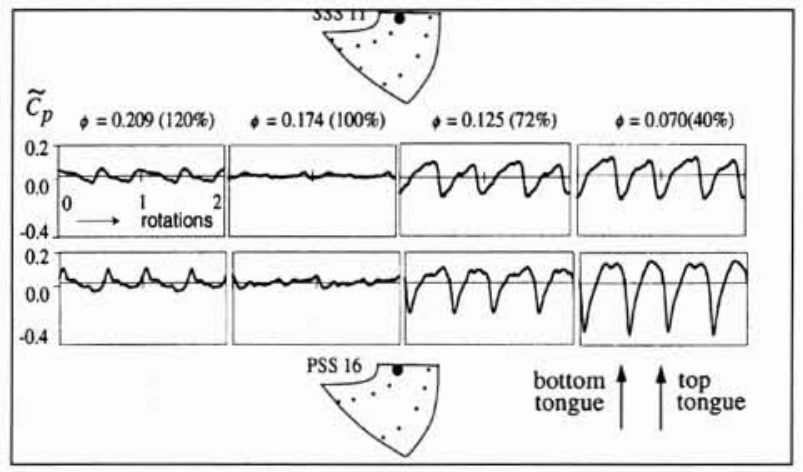

6. Phase averaged pressures for two transducers at 4 volume fluxes.

pressure field in the volute caused by the impeller rotation (i.e., vortex shedding).

Figure 6 reveals the phase averaged unsteady pressure for two transducers at 4 operating points over two rotations. The small static pressure head variation per rotation due to the horizontal alignment of the pump was subtracted. A tongue passing frequency was evident at a periodicity of twice per rotation. At bep load the pressure fluctuation in the impeller was small since the volute pressure distribution was uniform. At part load the volute steady pressure distribution $\left(C_{p}\right)$ rose between tongues, this manifests itself in the impeller as a rising pressure fluctuation after the blade passage rotated past a tongue (the arrows show radial alignment between the SSH trailing edge and the tongues). At overload this tendency was reversed as the volute flow had a decreasing pressure between volute tongues and thus a decreasing pressure fluctuation in the impeller. The blade passing frequency is not clearly evident but does weakly exist as a result of other blades rotating past the tongue. Of interest is the size of the pressure fluctuation, growing with sinking volume flux in part load to obtain magnitudes of $35 \%$ the pump head. Other authors [5] have obtained similar results with even greater fluctuation magnitudes being measured directly at the blade trailing edge.

It has previously been pointed out [Caignaert et al. 1991, Tourret et al. 1987] that pressure fluctuations within a pump impeller grow in magnitude as the volume flux is further removed from the bep and as the trailing edge $(s / L=1)$ of a

\section{NOMENCLATURE}

Symbols

$B$ impeller height

$D$ diameter

$P$ pressure

$u_{2}$ impeller outlet tip velocity

$\phi^{2} \quad \mathrm{cu}_{2} / u_{2} \mid$ flow coefficient

$\Gamma \quad$ circulation

$v$ wave velocity

Subscripts

$i \quad i^{\text {th }}$ pressure location

Overscripts

$\sim$ unsteady quantities

Acronyms

PSH Pressure Side Hub distance

SSH Suction Side Shroud distance

bep best efficiency point

$\begin{array}{ll}c & \text { velocity vector } \\ f & \text { frequency } \\ r & \text { radius } \\ \rho & \text { fluid density } \\ \theta & \text { pump shaft angle } \\ \beta & \text { impeller outlet angle }\end{array}$

$C_{p} \quad$ pressure coefficient

$n^{p} \quad$ pump shaft harmonic

$s \quad$ distance along a wall streamline

$\psi \quad \Delta \mathrm{P}_{2-1} /\left(0.5 \rho u_{2}^{2}\right)$

$\varphi \quad$ phase angle

$\lambda$ wavelength

1 pump inlet

2

pump impeller outlet
— steady quantities

PSS Pressure Side Shroud distance

SSS Suction Side Shroud distance 


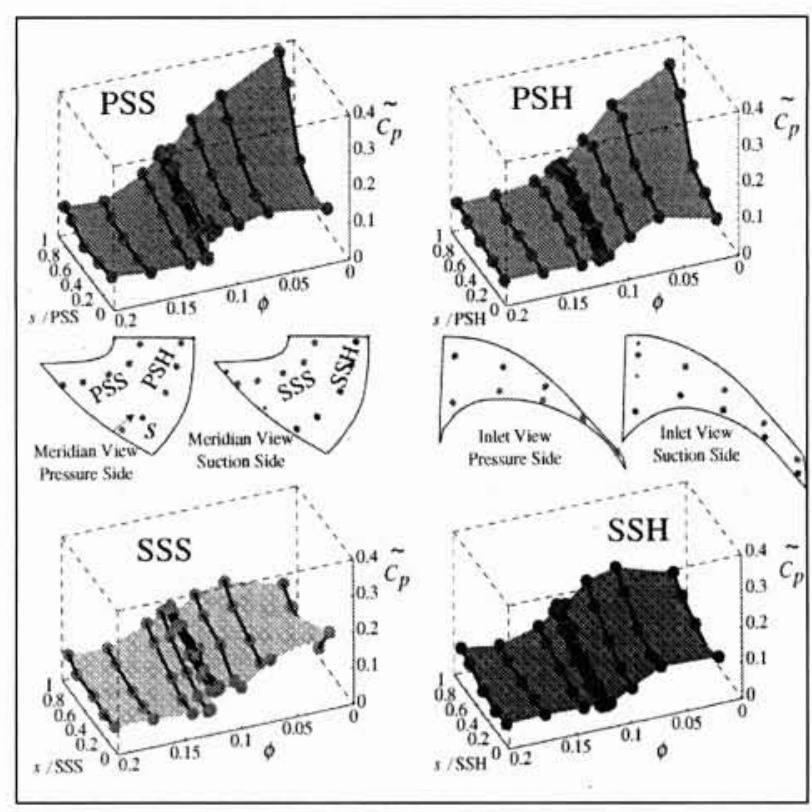

7. Pressure signal fluctuation on $\mathbf{4}$ wall streamlines.

blade was approached. This has also been found in this study shown in figure 7 where the $C_{p}$ is 2 standard deviations of a pressure signal magnitude. At $\phi=0.174(100 \%)$ on all four « wall streamlines » the magnitude was a minimum, expected from figure 5 where the volute flow circumferential pressure was most uniform. As the volume flux was removed from the best point the fluctuation magnitudes rose significantly. In general it can also be seen that the pressure fluctuation magnitude is greater on the pressure side of the blade channel than the suction side. On the suction side shroud (SSS) a greater amount of disorder seems to exist due to recirculation zones formation in the impeller [3].

The circumferentially distorted pressure distribution at the impeller outlet influenced the rotating impeller channel in a periodic fashion dominant at the tongue passing $(n=2)$ frequency. This can intuitively be verified from figure 6 . It has been quantitatively shown [8] that the tongue passing frequency $(n=2)$ has a fluctuation magnitude at least factor 5 greater than any other frequency present (i.e., $n=1, n=4$ ). This suggests presentation and interpretation of the phase information at the tongue passing harmonic.

\section{$V$ PHASE RELATIONS. ROTATING SYS- TEM}

The phase information along the 4 wall streamlines are all relative to a single pump shaft position meaning individual phase differences between pressure transducers may directly be interpreted as time lags or leads. Figure 8 reveals the processed phase information from FFTs for the tongue passing harmonic along the four wall streamlines within the impeller blade passage for 13 volume fluxes. The zero phase position occurs when the blade passage suction side hub trailing edge was radially aligned with the top tongue.

Observations for the phase measurements are presented in table 1.

Interpretation of the phase is made by considering a mathematical solution to the planar wave equation [French 1971] for the $n^{\text {th }}$ harmonic within a homogeneous region containing no sources written as,

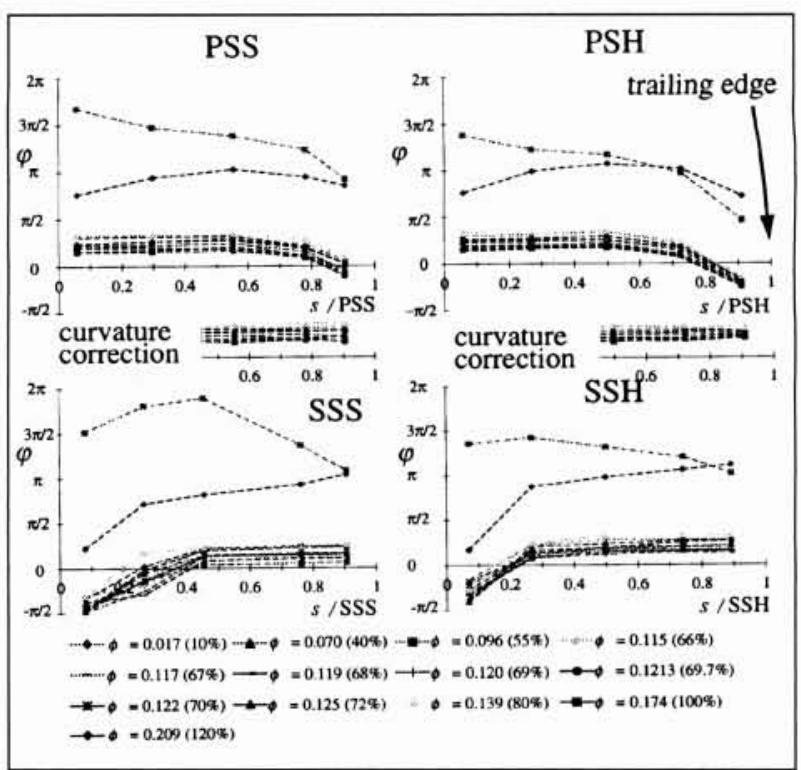

8. The phase information for the tongue passing $(n=2)$ harmonic along the 4 wall streamlines.

\begin{tabular}{|l|l|}
\hline$\phi \geq \phi_{\text {bep }}$ & no clear trend but $C_{p}$ magnitudes small \\
\hline & $\begin{array}{l}\text { PSS, PSH } \\
- \text { first } 3 \text { transducers have no phase } \\
\quad \text { change }\end{array}$ \\
& $\begin{array}{l}\text { - last } 2 \text { have phase lag from blade } \\
\text { curvature }\end{array}$ \\
& $\begin{array}{l}\text { SSH, SSS } \\
- \text { first } 2 \text { transducers have phase lead }\end{array}$ \\
& - last 3 have no phase change
\end{tabular}

Table 1. - Observations for the phase measurements in rotating system.

$$
\begin{aligned}
& \text { where } \begin{aligned}
\tilde{C}_{p p}(s, t) & =\left|\tilde{C}_{p n}\right| \sin \left(2 \pi s / \lambda_{n}+\varphi\right) \cos \left(\omega_{n} t\right) \\
\tilde{C}_{p}(s, t) & =\sum \tilde{C}_{p n}(s, t) .
\end{aligned}
\end{aligned}
$$

The phase shift in distance is chosen because the pressure transducers are at different spatial locations concurrently in time, the $\varphi$ varies with position $s$. Separating this equation to reveal the classic right $(R)$ and left $(L)$ moving waves provides,

$$
\tilde{C}_{p n}(s, t)=\left|\tilde{C}_{p n L}\right| \sin \left[\frac{2 \pi}{\lambda_{n}}(s-v t)+\varphi\right]+\left|\tilde{C}_{p n R}\right| \sin \left[\frac{2 \pi}{\lambda_{n}}(s+v t)+\varphi\right]
$$

where the wave velocity $v=w \omega_{n} \lambda_{n} / 2 \pi$. This is further reduced to the form,

$$
\tilde{C}_{p n}(s, t)=\left|\tilde{C}_{p n R, L}\right| \sin \left[ \pm 2 \pi f_{n} t+2 \pi s / \lambda_{n}+\varphi\right]
$$

The terms are interpreted as,

1) negative, right moving pressure wave (increasing s) downstream in impeller fluid flow,

2) positive, left moving pressure wave (decreasing s) upstream in impeller fluid flow.

In this manner changes in the phase with position in both the upstream and downstream moving waves in equation (2) are interpreted as,

1) $\mathrm{d} \varphi / \mathrm{d} s>0$, upstream (decreasing s) moving wave,

2) $\mathrm{d} \varphi / \mathrm{d} s<<0$, downstream (increasing s) moving wave. 
External noise and wave dispersion has been neglected in the above phase considerations. They are evaluated [Stegen \& Van Atta 1969] for two transducer signals $x$ and $y$ with the coherence and phase lead defined respectively as,

$$
\begin{aligned}
& n_{x y}^{2}(f)=S_{x y}(f) S_{x y}^{*}(f) / S_{x}(f) S_{y}(f) \\
& \varphi_{x y}(f)=-\tan ^{-1}\left[Q_{x y}(f) / C_{x y}(f)\right]
\end{aligned}
$$

where $S$ represents the cross spectral density with $C_{x y}$ and $Q_{x y}$ as the co-and quadrature spectrum. The coherence is interpreted as a frequency dependent correlation coefficient. The time delay and wave velocity, as in equation (1), between two transducers may also be obtained using,

$$
\begin{aligned}
\Delta t & =\varphi_{x x} f / 2 \pi f \\
v(f) & =2 \pi f s / \varphi_{x y}(f)
\end{aligned}
$$

The relative phase in equation (3) provides the same results as the FFT results in figure 8 but with no fixed zero reference phase (i.e., relative phases are determined). The concept of coherence is also introduced. The coherence between all transducers in figure 8 was high $(>0.93)$ at all volume fluxes $\phi<\phi_{b e p}$ indicating little disturbance in the form of noise and nonlinearities, the wave was nondispersive.

The transducers undergoing small phase changes $(\Delta \varphi<0.05)$ are classified as experiencing an acoustic wave in the blade passage since the acoustic wave propagation speed in water was measured to be $1326 \pm 20 \mathrm{~m} / \mathrm{s}$ meaning

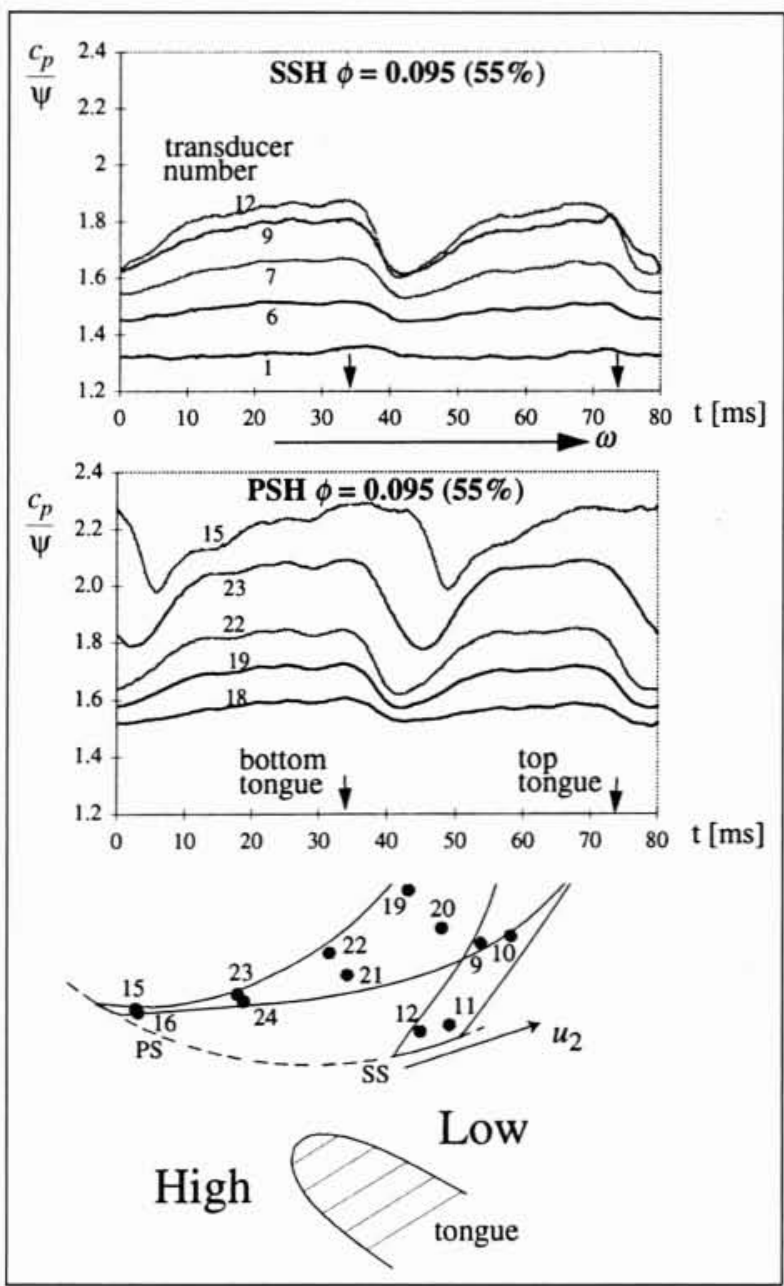

9. Two time domain phase averaged signals and a sketch of the blade passage as it moves past a volute tongue. pressure transducers and thus blade loading are influenced simultaneously. Both the pump shaft harmonic $(n=1)$ and twice the tongue harmonic $(n=4)$ have also been analyzed to reveal similar, not shown here, phase relations confirming the nondispersive nature of wave. The wave group and phase velocity are both equal to the acoustic velocity. Near the trailing edge $(s / \mathrm{PSH}=1, s / \mathrm{PSS}=1)$ however, $\mathrm{d} \varphi / \mathrm{d} s<0$ exists because these transducers arrive at the tongue later due to impeller blade curvature ; they experience the change in the pressure field across the tongue at a later time meaning a phase lag.

To further clarify the above explanation figure 9 depicts two time domain phase averaged pressure signals in part load with a two dimensional sketch of the blade passage. The arrows at bottom tongue and top tongue represent times when the blade passage suction side hub was aligned with a tongue. Examining the interaction with bottom tongue at time $30 \mathrm{~ms}$ the suction side of the blade passage was approaching the tongue and was influenced by the pressure in region High. As the blade suction side hub passed the bottom tongue $(34 \mathrm{~ms})$ it came under the influence of region Low having a lower pressure than region High because the volute was operating in part load. As seen in figure 9, the entire suction side was immediately influenced by this change in pressure at the blade passage outlet and blade pressures react instantaneously, at acoustic velocity. However, the blade passage pressure side was now under the influence of the pressure in region High and Low. More specifically the first three pressure transducers $(18,19,22)$ on the pressure side inlet react to Low while the last two transducers $(15,23)$ near the trailing edge were still, due to blade curvature, in High's influence. As the blade passage continued to rotate these last two transducers moved past the tongue coming into Low's influence. This blade curvature effect is geometrically removed from the phase information of the last 3 pressure side transducers shown in figure 8 labelled "curvature correction " to demonstrate the phase lags occurrence. This simplistic interpretation provides an understanding of the impeller-volute interaction physics represented in the pressure signal phase evaluations and an appreciation for the unsteady flow field during a blade passage - volute tongue interaction.

\section{CIRCULATION CONSIDERATIONS}

The implications of this acoustic pressure wave in the impeller can be realized from simple two dimensional classical aeroacoustics [Goldstein 1977]. Consider the starting transient vortex formation on an impeller blade impulsively accelerated from rest to some definite velocity (fig. 10a).

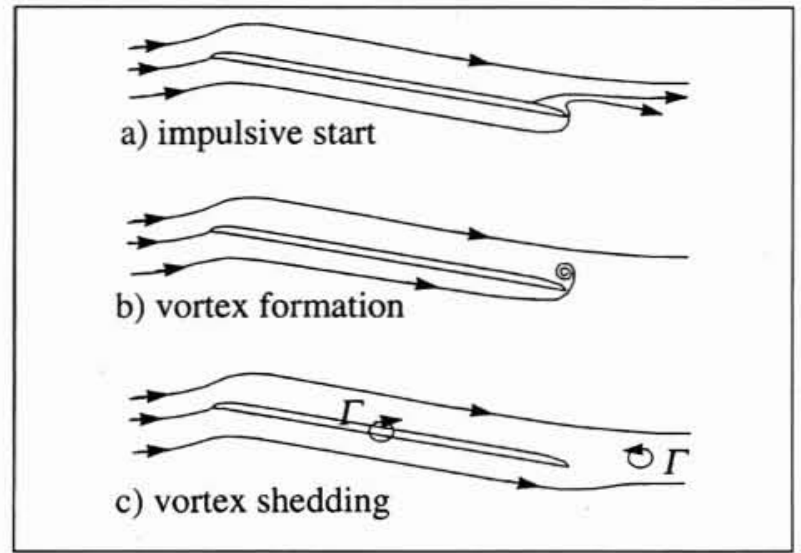

10. Transient vortex formation. 
The main flow shall be taken as inviscid, the boundary layer viscid. Kelvin's theorem states for an inviscid incompressible flow the circulation about the blade and surrounding fluid must at all times remain constant, $\mathrm{d} \Gamma / \mathrm{d} t=0$. In the transient acceleration the action of viscosity causes the formation of a thin boundary layer on the blade surface. Experimental observations [JSME 1988, Freymuth 1985] reveal high velocities at the blade trailing edge creating a low pressure region while at the rear stagnation point high pressure forms. This pressure gradient across the trailing edge causes separation and formation of a trailing edge vortex (fig. $10 \mathrm{~b}$ ). This vortex induces (i.e., according to Kelvin's theory) a circulatory flow about the blade which shifts the rear stagnation point to the trailing edge eliminating the trailing edge pressure gradient. The vortex formed then leaves the blade surface and is swept downstream (fig. 10c) to infinity. This behavior is a description of the classic Kutta-Joukowski condition. The circulation about the blade $\Gamma$ is proportional to the blade lift also in the unsteady case [Theodorsen 1939].

In the case of the pump impeller blade passage the acoustic pressure wave travelling upstream from the impellervolute interaction changes the pressure distribution instantaneously over the impeller blade suction side or pressure side which changes the blade lift. The instantaneous change in the blade lift requires a reaction from the circulation about the blade and thus the simplified case in figure 10 occurs with a periodicity. A continuous trail of vorticity creating a vortex wake must be formed, strength satisfying the Kutta-Joukowski condition. Since the volute pressure dominates the unsteady blade pressures at the $n=2$ harmonic it is conceivable (it has never been documented) that a synchronization between vortex wake frequency and the $n=2$ harmonic would exist. The fact that the blade outlet pressure is unsteady further complicates matters beyond (fig. 10) regarding the formation of the vortex wake. The acoustic propagation upstream in the blade passage thus induces a changing circulatory flow about the impeller blade which in turn changes the circulation in the downstream flow. This is termed here a circulatory flow effect.

\section{VII $\square$ CIRCULATION EFFECT. STATIO- NARY SYSTEM}

As the impeller outlet flow travels downstream its structure decays in strength and changes in form. Presented in figure 11 are the phase averaged unsteady total pressures behind the impeller at mid passage height for $\phi=0.174$ $(100 \%)$. They were measured using a 1 hole fast response probe [12]. These measurements assume an outlet flow circumferential symmetry in the wake formation and evolution permitting the blade passage phase averaging. There are 10 radial positions shown over one rotation of the impeller. The distinct phenomena of a viscous region and a circulation induced peak [Yuasa \& Hinata 1979] are identified. The circulation peak propagates a pressure disturbance at acoustic velocity decaying with increased radial distance. This peak is due to the rotation of the blades past a stationary observer. The blade pressure phase change $(n=2)$ dictates a changing blade circulation which when moved induces an unsteady flow field at acoustic velocity according to the hydroacoustic form of the Biot-Savat law,

$$
c(x)=\frac{1}{4 \pi} \oint \Gamma \frac{x \times d I}{r^{3}}
$$

where $\mathrm{d} l$ is an incremental length along any given vortex line. It reveals how an unsteady flow field may be induced, as measured in figure 11 , from a moving circulation (i.e., $r$ varies). Important to recall is the purely kinematic nature of this relation meaning it is valid in both inviscid and viscous flows. In the far field the circulation peak is registered as rotor acoustic. The more dominant effect in the near field is viscous from the trailing edge wake. The viscous region having an outlet flow angle between $30^{\circ}$ to $80^{\circ}, \beta_{2}$ blade outlet is $30^{\circ}$, becomes wider and decreases with amplitude as the radius increases, dissipating more slowly initially than the circulation peak.

\section{VIII — CONCLUSIONS}

The circumferential pressure variation within the volutes of centrifugal pumps resulting from a mismatch of angular momentum exchange is well documented in the literature. This pressure variation was experienced in the rotating system as unsteady, dominated by the tongue passing frequency.

The volute tongue acts as a boundary separating two distinctly different flow regimes providing a pressure gradient responsible for an abrupt flow field fluctuation in the impeller. The magnitude of the flow field unsteadiness grew as the volume flux was further removed from the bep volume flux, particularly below the flow coefficient at which pump characteristic hysteresis occurs. In deep part load the pressure

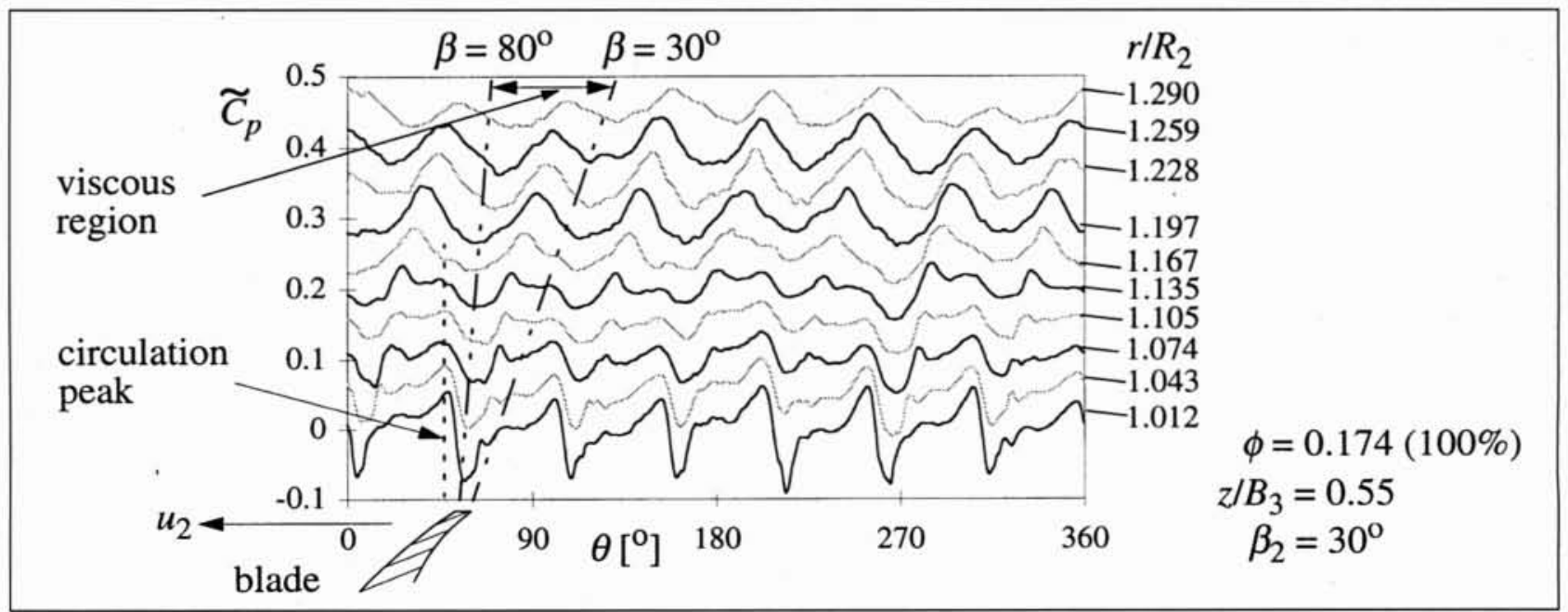

11. Flow evolution downstream near impeller outlet mid height. Viscous and circulation induced unsteady total pressure are quantified. 
field fluctuations grew to the $35 \%$ of the pump head. The pressure fluctuation at the impeller outlet propagated upstream through the blade passage at acoustic velocity excluding those locations where blade curvature and rotation provided a phase lag. The concept of circulation was implemented to physically interpret the influence of impeller pressure field unsteadiness.

\section{REFERENCES}

[1] ISO 1987: 5198, International Organization for Standardization, Centrifugal, mixed flow and axial pumps - Code for hydraulic performance test - Precision grade, Edition 1.

[2] KaUPerT K.A. (1997). - The Unsteady Flow Field in High Specific Speed Radial Pumps, ETH Zurich Dissertation \#12068.

[3] Kaupert K.A., Holbein P. \& Staubli T. (1996), - A First Analysis of Flow Field Hysteresis in a Centrifugal Impeller. J. Fluids Eng., Vol. 118, Dec.

[4] LORETT J.A. \& GopalakrishNAN S. (1986). - Interaction Between Impeller and Volute of Pumps at Off-Design Conditions. J. Fluids Eng., Vol. 108, p. 12-18.

[5] AdKins D.R. \& BRENNEN C.E. (1988). - Analyses of hydrodynamic radial forces on centrifugal pump impellers. ASM J. Fluids Eng., Vol. 110, p. 20-28.

[6] WeSCHE W. (1987). - Messungen am Spiralgehäuse einer Kreiselpumpe, Technische Rundschau Sulzer 2/87, p. 17-20.
[7] Tourret J., Kamga M., Foucher D. \& Kermarec J. (1989). - Hydraulic noise studies from a centrifugal pump through pressure pulsations measurements in the volute and in the impeller, Société Hydrotechnique de France, $\mathrm{XX}^{\mathrm{e}}$ Journées de 1'Hydraulique, Lyon, 4-6 April.

[8] KAUPERT K.A. \& Staubli T. (1997). - The Influence of the Volute on the Pressure Field within a High Specific Speed Centrifugal Pump Impeller, JSME Centennial Grand Congress on Fluid Engineering, Tokyo, Japan, July 13-16.

[9] ARNDT N.K.E. (1988), - Experimental Investigation of Rotor-Stator Interaction in Diffuser Pumps Dissertation California Institute of Technology, Eng. \& App. Sci.

[10] BREnNen C.E. (1994), - Hydrodynamics of Pumps Concepts ETI and Oxford University Press.

[11] Makay E. (1988). - Power Plant Pump Trouble-Shooting State of the Art Overview, EPRI Report CS-5857, p. 4.14.23 .

[12] Staubli T., Holbein P. \& Kaupert K.A. (1995). - Verification of Computed Flow Fields in a Pump of High Specific Speed, International Symposium on Numerical Simulations in Turbomachinery, ASME-JSME Fluids Eng. Conf., S.C. USA, August, No. FED95-NSTM-AH9.

[13] Tamatsukuri T., Kikuchi K. \& Satch J. (1992), - Progress with Ultra High Head Single-Stage Pump-Turbines, Water Power \& Dam Construction, Dec., p. 29-33.

[14] Van den Braembussche R.A. \& Senn M.Th. (1989). Influence of a circumferential exit pressure distortion on the flow in an impeller and diffuser. Société Hydrotechnique de France, $\mathrm{XX}^{\mathrm{e}}$ Journées de 1'Hydraulique, Lyon, 4-6 April, Report No. 12. 